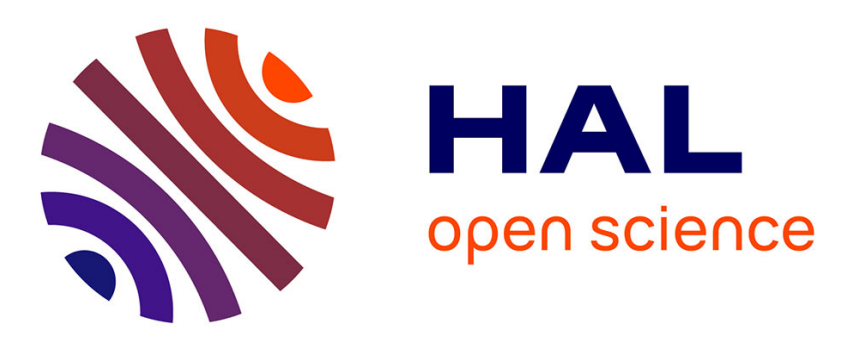

\title{
LES PROGRAMMES EXPERIMENTAUX AUPRES DU SYNCHROCYCLOTRON D'ORSAY
}

\author{
L. Bimbot
}

\section{To cite this version:}

L. Bimbot. LES PROGRAMMES EXPERIMENTAUX AUPRES DU SYNCHROCYCLOTRON

D'ORSAY. Congres General de la Societe Francaise de Physique, 1979, Toulouse, France. pp.C3155-C3-160, 10.1051/jphyscol:1980322 . jpa-00219841

\section{HAL Id: jpa-00219841 https://hal.science/jpa-00219841}

Submitted on 1 Jan 1980

HAL is a multi-disciplinary open access archive for the deposit and dissemination of scientific research documents, whether they are published or not. The documents may come from teaching and research institutions in France or abroad, or from public or private research centers.
L'archive ouverte pluridisciplinaire HAL, est destinée au dépôt et à la diffusion de documents scientifiques de niveau recherche, publiés ou non, émanant des établissements d'enseignement et de recherche français ou étrangers, des laboratoires publics ou privés. 


\title{
LES PROGRAMMES EXPERIMENTAUX AUPRES DU SYNCHROCYCLOTRON D'ORSAY
}

\author{
L. Bimbot \\ Institut de Physique Hucléaire, B.P. no 1,91406 Orsay, France.
}

\begin{abstract}
Résumé.- Les programmes expérimentaux auprès du S.C. d'orsay ont commencé il y a un an environ. L'accélérateur est une machine stable mais présentant quelques défauts de jeunesse. De nombreuses voies de recherches amorcées avec un certain succès sont décrites ci-dessous.
\end{abstract}

Abstract.- A brief review of experimental works undertaken with the orsay S.C. beams is given. Most of them have provided preliminary encouraging results. Youth defects of the accelerator have now to be suppressed.

Trois comités d'expêriences se sont đejjà réunis pour examiner les demandes d'expériences auprès du S.C. d'orsay. Après I'exposé des lettres d'intention en décembre 76 , des priorités ont été données lors des deux autres réunions (octobre 77 et janvier 79). Les demandes examinées en janvier 79 émanaient de 88 chercheurs pour 2640 heures de faisceau. Il $y$ avait 30 chercheurs de I'IPN, 12 du CSNSM et 46 d'autres laboratoires, de province ou de l'étranger. L'exposé ci-dessous est tiré en partie de ces réunions et des êlements plus récents que chacun a bien voulu me communiquer. Je vous prie de m'excuser pour tout oubli éventuel ou interprétation erronée.

Après avoir rappelé les caractêristiques techniques actuelles du synchrocyclotron et des salles d'expérience (construites et équipées par I'IPN) et au séparateur en ligne ISOCELE II, des lignes et de la salle de physique (construits en commun par l'IPN et le CSNSM), je ferai le point sur les résultats obtenus d"une part en spectroscopie en ligne et d'autre part en étude des réactions nucléaires en dégageant les premiers rêsultats et les đéveloppements prévus.

1. LES INSTALIATIONS ACTUELLES.- 1.1. L'accélérateur 11/.- Les essais du S.C. se sont répartis entre juin 77 et mars 78 . Les temps d'essais avec faisceau ont étê courts par rapport au temps des travaux de finition de transformation. Les caractéristiques actuelles sont présentées dans le tableau I auquel il convient d'ajouter la remarque suivante : le rendement $d$ 'extraction est la valeur mesurée mais cela ne signifie pas qu'un faisceau de 1,2 à $2,4 \mu \mathrm{A}$ soit utilisable en sortie du s.C. L'intensité maximum utilisable sur les lignes de faisceau est de $0,6 \mu \mathrm{A}$ en protons. Cette limitation est due aux problèmes suivants non encore totalement résolus :

- perte interne à la machine (sur le Dee),

- perte sur les éléments de transport de faisceau,

- épaisseur des murs de protection du bâtiment.

Tableau I

Caractéristiques actuelles du synchrocyclotron d'orsay

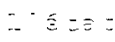

\begin{tabular}{|c|c|c|c|c|c|c|c|}
\hline \multirow{2}{*}{$\begin{array}{l}\text { PARAMETRES SC } \\
\text { PARTICULES }\end{array}$} & \multicolumn{7}{|c|}{ 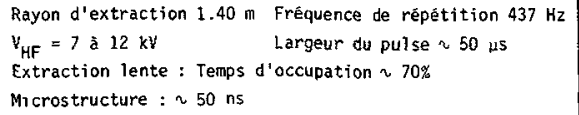 } \\
\hline & $\mathrm{p}$ & & & & & 4 & \\
\hline ENERGIES en MeV & 200 & 90 & 108 & 238 & 282 & 182 & 217 \\
\hline INTENSITE INTERNE & \multicolumn{7}{|l|}{2 มे $3 \mu \mathrm{A}$} \\
\hline $\begin{array}{l}\text { RENDEMENT } \\
\text { EXTRACTION }\end{array}$ & \multicolumn{7}{|c|}{$\begin{array}{l}60 \text { à } 80 \% \text { en extraction rapide } \\
40 \text { a } 60 \% \text { en extraction lente }\end{array}$} \\
\hline
\end{tabular}

L'exploitation dans ces conditions en 1978 et 1979 estrésumée sur la figure 1. De novembre 78 à mai 79 , le "trou" d"exploitation correspond à des anomalies de fonctionnement du CeM et de la $\mathrm{HF}$ et à des pannes diverses, des finitions, de mises au point. Le temps accordé à la physique doit être pondéré par un facteur d'efficacité de $50 \%$ environ, correspondant à la mise au point des faisceaux et des systèmes de détection d'une part, et au fonctionnement haché du s.c. dans cette période de jeunesse. Les quatre types de particules sont utilisés mais principalement à énergie haute. La répartition entre les différentes salles est donnée figure 1.

1.2. Les salles pour étude des réactions nucléaires .- L'implantation auprès du S.C. est donnée figure 
2 et les caractéristiques des faisceaux sont données dans le tableau II.

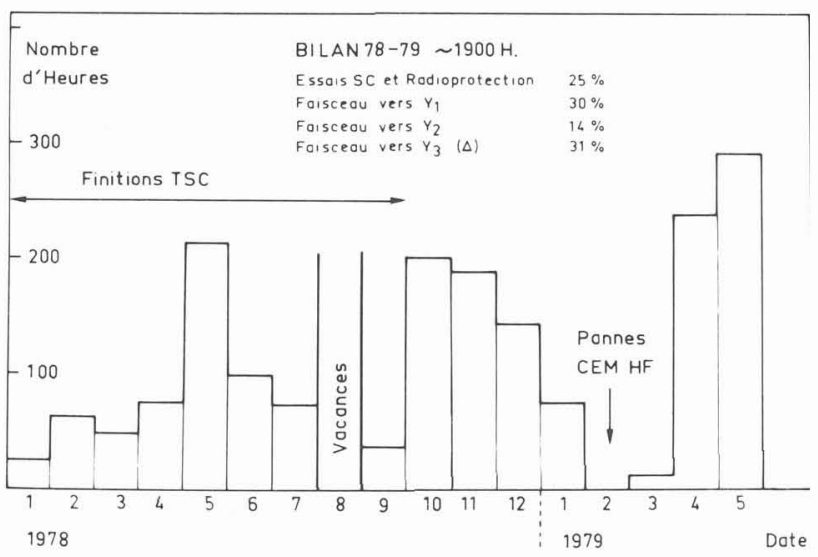

Fig. 1.- Résumé de l'exploitation du S.C. en 1978 et 1979

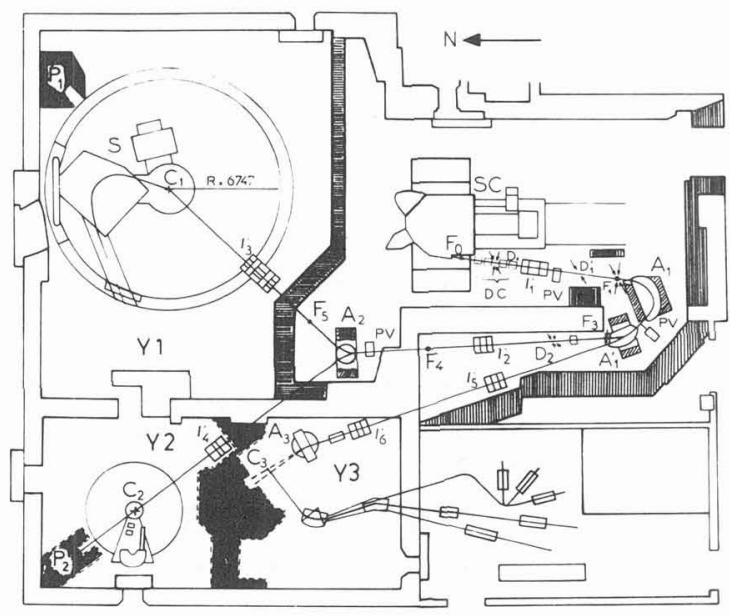

Fig. 2.- Implantation générale auprès du S.C.

Tableau II

Caractéristiques actuelles du faisceau dans les salles d'expérience.

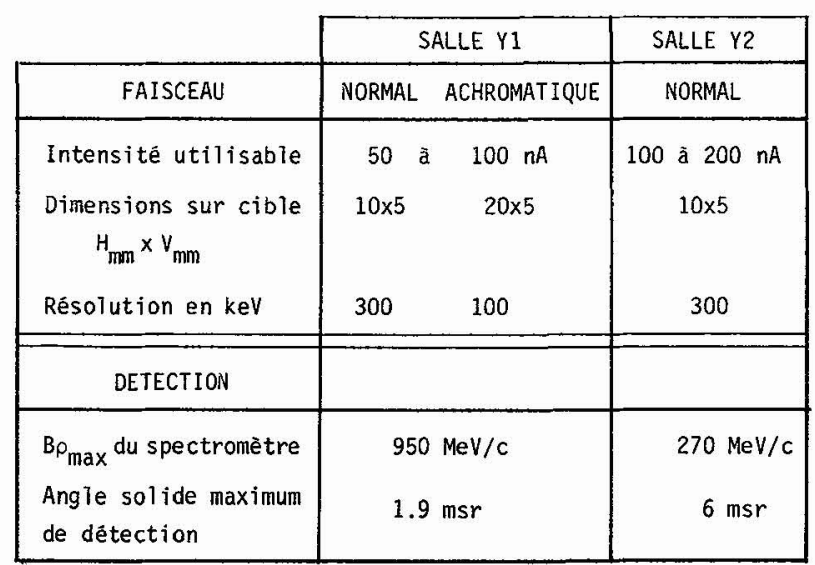

La salle $\mathrm{Y} 1$ est équipée d'un grand spectromètre "MONTPELLIER" suivi d'une détection performante à base de 2 chambres à fils à lecture du baxycentre des charges sur la cathode déclenchée par un "trigger" de scintillateurs et photomultiplicateurs permettant des mesures à fort taux de comptage et bonne résolution spatiale pour un grand éventail de particules $/ 2 /$.

L'acquisition en ligne sur un calculateur HP $21 \mathrm{MXE}$ et un programme de reconstruction de trajectoires et projection sur le plan focal, permettent d'atteindre une résolution optimale $/ 3 /$.

La salle Y2 est équipée du même système de détection par chambres à fils derrière un spectromètre "MATHUSALEM" de faible Bp ( $\leqslant 270 \mathrm{MeV} / \mathrm{C})$. I'angle solide est augmenté par $1^{\prime}$ utilisation d'un doublet de lentilles quadrupolaires placé entre la cible et le spectromètre et focalisant les particules détectées sur un petit scintillateur A placé à l'entrée de l'aimant. Trois scintillateurs B, C et D servent à identifier les particules à la sortie. Un temps de vol est pris entre $A$ et $B$ d'une part, et entre $B$ et D d'autre part, pour augmenter le taux de réjection des évẽnements parasites. La mesure des sections efficaces inféxieures au nb est ainsi espérée.

1.3. Le séparateur en Iigne ISOCELE II /4/.- Entièrement remanié, le séparateur d'isotopes en ligne ISOCELE I est devenu ISOCELE II avec un certain succès, Les premières comparaisons laissent espérer un gain de 5 à 10 dans la production de certains éléments. Cela provient essentiellement d'une amélioration de la source et du système de transport des ions.

Trois lignes d'expériences sont disponibles. Ia ligne 1 a êté munie, dans un premier temps, d'un dispositif de spectroscopie $\alpha, \beta$ et $\gamma$. Sur la ligne 2, en plus d'un dispositif de spectroscopie $\alpha, \beta$ et $\gamma$ a été installê un spectrographe à $180^{\circ}$ pour la mesure à haute résolution des électrons de basse énergie. La ligne 3 est équipée d'un spectromètre Gerholm destiné aux mesures de vie moyenne. Un système très performant d'acquisition de données a été mis au point à I'IPN. Il gère les voies directes et les voies corrélées (jusqu'à 8 voies) à partir d'instructions spécifiques enregistrées par I'utilisateur dans des mémoires protégées. L'enregistrement se fait sur deux dérouleurs de bandes magnétiques organisés en ping-pong ou en sélection. L'achat de plusieurs Ge intrinsèque est en cours pour permettre des mesures rapides de corrélations 
angulaires.

Le tableau III résume 1'utilisation du séparateur depuis sa mise en route $/ 5 /$. d'étude de transitions Ho $\rightarrow$ Dy avec cette cible. La difféxence de volatilité entre Ho et $\mathrm{Tb}$ n'est pas très grande mais suffit cependant pour une production

Tableau III

Répartition et utilisation des faisceaux du S.C. vers ISOCELE II

\begin{tabular}{|c|c|c|c|c|c|c|}
\hline \multicolumn{2}{|c|}{$\begin{array}{c}\text { DATES } \\
\text { Mois - Annëe }\end{array}$} & PARTICULES & $\begin{array}{c}\text { INTENS ITES } \\
\text { nA }\end{array}$ & $\begin{array}{l}\text { DUREES } \\
\text { Heures }\end{array}$ & CIBLES & BUTS POURSUIVIS \\
\hline 04 & 78 & $\mathrm{D}, \mathrm{F},{ }^{3} \mathrm{He}$ & 20 à 100 & 12 & - & {$\left[\begin{array}{l}\text { Faisceaulogie } \mathrm{Sc} \text { - Isocèle } \\
\text { Mesures de radioprotection }\end{array}\right.$} \\
\hline 05 & 78 & $\mathrm{P},{ }^{3} \mathrm{He}$ & 90 à 150 & 78 & $\mathrm{Au} ; \mathrm{Gd} ; \mathrm{Er}$ & [Tests Isocèle II \\
\hline 06 & 78 & $\mathrm{P},{ }^{3} \mathrm{He}$ & 60 à 300 & 53 & $\mathrm{Ba} ; \mathrm{Nd} ; \mathrm{Au}$ & $\begin{array}{l}\text { Mesures de production } \\
\text { Tests de cibles }\end{array}$ \\
\hline 07 & 78 & $\mathrm{P},{ }^{3} \mathrm{He}$ & 90 à 100 & 28 & $\mathrm{Ag} ; \mathrm{Gd}$ & Expériences de physique \\
\hline 10 & 78 & $\mathrm{P}$ & 60 à 150 & 70 & $\mathrm{Gd} ; \mathrm{Au} ; \mathrm{Pr}$ & Essais de cibles \\
\hline 11 & 78 & $P$ & 150 à 200 & 44 & $\mathrm{Er} ; \mathrm{Au}$ & Productions \\
\hline 12 & 78 & ${ }^{3} \mathrm{He},{ }^{4} \mathrm{He}$ & 100 à 600 & 35 & Sn & Utilisation du spectromètre plat \\
\hline 04 & 79 & $P$ & 300 & 14 & $\mathrm{Au}$ & $\begin{array}{l}\text { Essais de cibles } \\
\text { Productions }\end{array}$ \\
\hline 05 & 79 & ${ }^{3} \mathrm{He}$ & 600 & 48 & $\mathrm{~Tb}$ & Expériences de physique \\
\hline
\end{tabular}

2. I.ES PREMIERS RESULTATS.- 2.1. En spectroscopie de ligne.- De nombreux tests de cibles et de production ont déjà eu lieu. Il est impossible de tout présenter dans ce compte rendu et je me bornerai à quelques exemples de résultats marquants. Toute information supplémentaire peut être obtenue auprès de $M$. Berg $/ 6 /$.

- Mesures de vies moyennes /7/.- Les premières études ont concerné le ${ }^{143} \mathrm{Sm}$ et $11188_{\mathrm{Au}}$. Les mesures sont faites avec le spectromètre Gerholm. La vie moyenne du premier niveau excité du ${ }^{143} \mathrm{Sm}$ a été mesurée. Le résultat : $690 \pm 50$ ps, montre que la transition $1 / 2^{+} \rightarrow 3 / 2^{+}$est beaucoup plus accélérée que la transition correspondante dans le ${ }^{141} \mathrm{Nd}$ ce qu'il est difficile de concilier avec l'interprétation de l'état $1 / 2+$ de $14{ }^{1} \mathrm{Sm}$ comme état pur à un trou. Les études des niveaux de $188_{\text {Au }}$ peuplés par la désintégration de ${ }^{188} \mathrm{Hg}$ ont été poursuivies; les retards entre les transitions de $190 \mathrm{keV}$ et de 115 $\mathrm{keV}(240 \pm 20 \mathrm{ps})$ et entre les transitions de $67 \mathrm{keV}$ et de $16 \mathrm{keV}(1,1+0,3 \mathrm{~ns})$ ont été mesurés.

- Etude des transitions но $\rightarrow$ Dy /8/.- La figure 3 présente les courbes des productions les plus récentes établies à partir d'une cible de terbium métal irradié en ${ }^{3} \mathrm{He}$. Ce sont les moyennes des points expérimentaux qui fluctuent largement autour des courbes. Ces essais rentrent dans un programme utilisable.

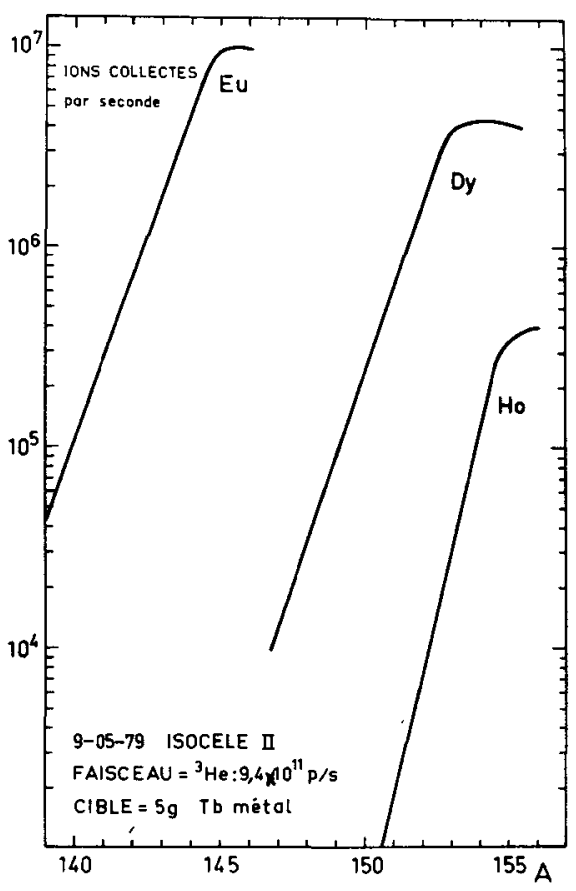

Fig. 3.- Courbes de production obtenues par irradiation de terbium métal par des ${ }^{3}$ He.

- Recherche de 1102 In /9/.- Lá figure 4 donne les courbes comparées des productions obtenues par irradiation d'une cible d'étain par des ${ }^{3}$ He et des 
${ }^{4} \mathrm{He}$. Les valeurs sont trop faibles pour autoriser 1 'étude envisagée du ${ }^{102} \mathrm{Ca}$ par production de ${ }^{102} \mathrm{In}$. Les plus fortes productions en ${ }^{3} \mathrm{He}$ laissent esperer que 1'ixradiation par protons donnera un gain d'un facteur 10 qui renorait cette expérience réalisable.

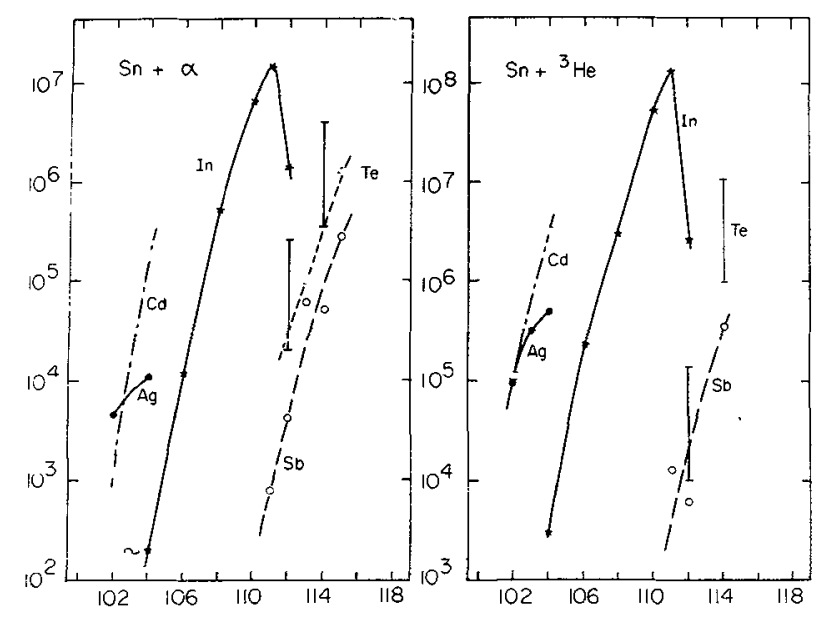

Fig. 4.- Courbes de production obtenues par irradiation d'étain par des ${ }^{3}$ He et des ${ }^{4}$ He.

\subsection{En étude des mécanismes de réaction.-}

- Expériences de production de pions /10/.I'étude de la production de pions au voisinage du seuil se fait auprès du spectromètre MATHUSALEM spécialement équipé à cet effet. Les mesures $\left(p, \pi^{+}\right)$ont été faites avec une cible de ${ }^{10_{B}}$ dans un premiex temps et le spectre présenté figure 5 donne les premiers niveaux excités observés du ${ }^{11} \mathrm{~B}$. Une expérience est prévue avec une cible liquide $d^{\prime}{ }^{3} \mathrm{He}$. Les distributions angulaires sont possibles entre $15^{\circ}$ et $165^{\circ}$ laboratoire. Ces expériences font intervenir les composantes de grand moment dans les fonctions d'onde du noyau et pose le problème du mécanisme de réaction encore largement ouvert. Les résultats actuels ne sont que préliminaires. A $200 \mathrm{MeV}$, les mesures sont en bon accord avec celles obtenues sur le ${ }^{10} \mathrm{~B}$ par Piles et al. /11/:

$$
\left(\frac{d \sigma}{d \Omega}\right)_{25^{\circ} \mathrm{CM}} \simeq 1,12 \pm 0,07 \mu \mathrm{b} / \mathrm{sr}
$$

La recherche de production exclusive de $\pi^{+}$par reaction $\left({ }^{3} \mathrm{He}, \pi^{+}\right)$avec les ${ }^{3} \mathrm{He}$ de $282 \mathrm{MeV}$ est prévue pour un prochain avenir.

- Expériences de capture radiative de protons /12/.- De même nature que la production de pions mais avec une voie de sortie mieux adaptée aux calculs, ces expériences ont été proposêes auprès du S.C. Une expérience préliminaire a eu lieu par la réaction $d(p, \gamma)^{3} \mathrm{He}$ avec détection de $1^{13}$ He de recul en coincidence avec le $\gamma$ détecté dans un compteur Cerenkov. Les mesures sont en cours de dêpouillement ( I'expérience s'est déroulée en mai 79).

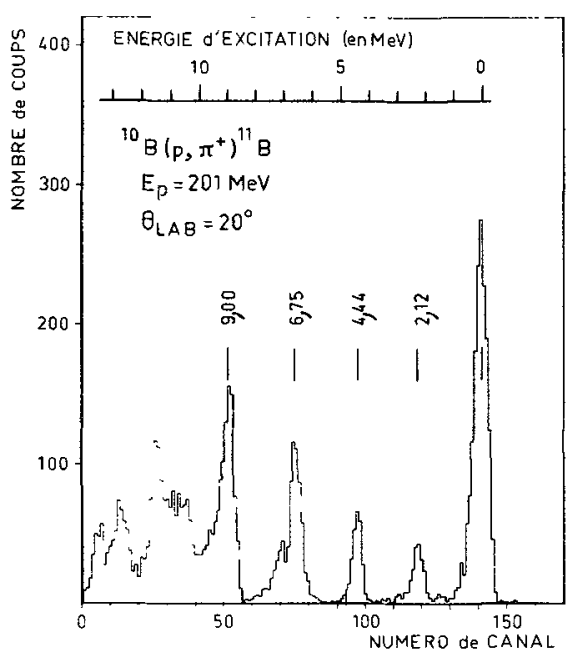

Fig. 5.- Spectre préliminaire de production de $\pi^{+}$

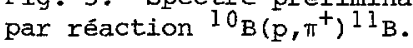

- Expériences $(\mathrm{p}, \mathrm{x} \gamma)$ en collaboration OrsayClermont Ferrand /13/.- Extension à $200 \mathrm{MeV}$ d'expériences fructueuses à $400 \mathrm{MeV}$, le but poursuivi est une meilleure connaissance des processus découlant de l'excitation importante du noyau provoquée par la diffusion d'un proton de moyenne énergie. Le premier stade de l'expérience a été la détection des $\gamma$ de faible énergie caractéristiques des désexcitations des différents états des noyaux obtenus à partix d'une cible d'aluminium. Les résultats préliminaires obtenus semblent montrer des différences sensibles par rapport à $400 \mathrm{MeV}$ donc 1 'accès à des informations nouvelles.

Ces mesures vont donc être affinēes avec la détection en coincidence du proton diffusé suivant des géométries particulières, avec l'aimant d'analyse ASTERIX equipé de chambres proportionnelles. I'implantation est en cours en salle Y1. Ces dispositifs doivent permettre de réaliser aussi des analyses en coinncidence du type $(p, 2 p \gamma)$ sur des noyaux légers et d'aborder le détail des mécanismes dans les quasi élastiques.

- Etude du break-up du deuton par réaction $d(p, p n) p / 14 / .-$ Entrant dans le cadre d'un programme d'étude d'interactions à deux, trois ou quatre nucléons dans le "break uF" de systèmes de trois ou quatre nucléons, I'expérience précédente s'est déroulée durant 48 heures. Le nouveau compteur à neutrons avec hodoscope pour la discrimination neutrongamma a fonctionné de manière satisfaisante. Les 
prolongements de I'expérience sont prểus pour 1980 .

\subsection{En étude de structure nucléaire.-}

- Etude des résonances géantes /15/.- L'étude de la région des résonances géantes a été faite par diffusion (d,d') à $108 \mathrm{MeV}$ sur $90 \mathrm{Zr}, 120 \mathrm{Sn}$ et $208 \mathrm{~Pb}$ dans un domaine angulaire $\left(4^{\circ}\right.$ à $\left.10^{\circ}\right)$ couvrant le minimum caractéristique de la résonance monopolaire. Pour les 3 noyaux, la résonance monopolaire a été aisément mise en évidence. Le rapport hauteur de résonance à hauteur du continuum est de l'orơre de 1 aux petits angles. Les principaux résultats sont donnés dans le tableau IV. On a pu extraire la valeur du coefficient de compressibilité du noyau A : $\mathrm{K}_{\mathrm{A}}=133 \pm 3 \mathrm{MeV}$.

\section{Tableau IV}

Paramètres mesurés par l'étude des résonances géantes par diffusion (D $\left.D^{\prime}\right)$.

\begin{tabular}{|c|c|c|c|c|c|}
\hline Noyau & $E_{0} \mathrm{MeV}$ & $\Gamma_{\mathrm{O}} \mathrm{MeV}$ & $\mathrm{K}_{\mathrm{A}} \mathrm{MeV}$ & $\mathrm{E}_{2} \mathrm{MeV}$ & $\Gamma_{2} \mathrm{MeV}$ \\
\hline${ }^{90_{\mathrm{Zr}}}$ & 17,2 & 4,3 & 130 & 14,1 & 4,0 \\
${ }^{120} \mathrm{Sn}$ & 16,1 & 3,0 & 135 & 13,2 & 3,2 \\
$208_{\mathrm{Pb}}$ & 13,5 & 2,8 & 133 & 10,5 & 2,8 \\
\hline
\end{tabular}

- Etude des états de couche profonde de neutron de grand moment orbital par réaction $\left({ }^{3} \mathrm{He}, \alpha\right)$ à 280 $\mathrm{MeV} / 16 /$.

Ces expériences demandent une bonne résolution et sont possibles grâce à la grande sélectivité des états de grand moment orbital de ces réactions de transfert. Des essais en réaction $(d, t)$ sur le $208 \mathrm{~Pb}$ se sont révélés encourageants et 1 'expérience $\left({ }^{3} \mathrm{He}, \alpha\right)$, non encore réalisée à cause de pannes au s.c., est programmée pour bientôt.

2.4. Autres expériences ayant êté commencées auprès du s.C. -

- Etude expérimentale de la production d'isotopes légers He, Ii, Be dans les intexactions $\alpha+\alpha$ à $220 \mathrm{MeV} / 17 /$.

Ces expériences présentent un grand intérêt sur le plan astrophysique pour l'interprétation de la composition du milieu interstellaire. La détection se fait au moyen de jonctions au silicium. La cible est gazeuse. Une première expérience a permis d'analyser l'ensemble du spectre d'énergie pour les charges 3 et 4 et la partie basse énergie pour les charges 2 . Les divers nuclides sont parfaitement identifiés.
Les premières évaluations ont montré que les sections efficaces de production de ${ }^{6} \mathrm{Li}-{ }^{7} \mathrm{Li}$ décroissent fortement avec l'énergie incidente, ce qui confirme le fait que les mécanismes de spallation sont insuffisants pour expliquer la valeur du rapport ${ }^{6} \mathrm{Li} /{ }^{7} \mathrm{Li}$ observé dans le milieu interstellaire.

- Etude de la détection des protons de haute énergie par un empilement de détecteurs Ge intrinsèque $/ 18 /$.

Le but physique poursuivi est 1 'étude des particules émises dans une large dynamique par bombardement de protons de moyenne énergie (170 MeV). Un télescope de 5 germanium intrinsẽque a êté réalisé par 1 'institut IKP du KFA de Jülich et essayé par deux fois auprès du s.C. La première fois en vision directe de la cible, pour identifier le plus grand nombre possible de particules et d'énergies. Il a été possible de détecter les protons de $165 \mathrm{MeV}$ avec une résolution de $470 \mathrm{keV}$. Un deuxième essai récent vient de se dérouler en plaçant ce télescope derrière le spectromètre MONTPELLIER pour étudier la réponse des détecteurs à différentes énergies de protons obtenues par diffusion élastique sur une cible de $\mathrm{CD}_{2}$ à différents angles.

- Etude du taux de formation des isotopes d'iode et de xénon à usage médical par réaction ${ }^{127}$ I $(d, 6 n) / 19 /$.

Les possibilitês de production de 1 'isotope ${ }^{123} \mathrm{I}$ à partix de la réaction $127 \mathrm{I}(\mathrm{d}, 6 \mathrm{n})^{123} \mathrm{Xe} \stackrel{2 \mathrm{H}}{\longrightarrow}{ }^{123} \mathrm{I}$ $\stackrel{12 \mathrm{H}}{\longrightarrow} 123 \mathrm{Te}$ (stable) ont été étudiées au s.c. Ce radioélément de période relativement "courte" par rapport à celle de ${ }^{131}$ I ( 8 jours) pourrait rapidement remplacer ce dexnier dans ses utilisations médicales en $\gamma$ scintigraphie principalement. Les taux de production atteints avec une intensitê de $10 \mathrm{nA}$ de deutons de $108 \mathrm{MeV}$ ralentis aux environs de $70 \mathrm{MeV}$ donnent $\mathrm{R} \geqslant 5,7 \mathrm{mC} / \mu \mathrm{A} / \mathrm{h}$ en ${ }^{123} \mathrm{I}$ avec un pourcentage de mélange de ${ }^{125}$ I de $0,26 \%$.

Une production systématique $d^{123}$ I à usage médical nécessiterait $2 \mathrm{H}$ d'irradiation en deutons, à une intensité $>1 \mu \mathrm{A}$ tous les quinze jours. C'est en cours d'étude à l'IPN mais les contraintes invoquées sont, poux le moment, incompatibles avec la fiabilité de la machine et les programmes expérimentaux de physique prévus.

3. LES DEVELOPPEMENTS.- Les programmes expérimentaux évoqués ci-dessus ne font que commencer et certains demandent I'accès aux caractéristiques finales du cahier des charges de la transformation du synchrocyclotron, ainsi qu'une finition donnant une grande 
fiabilité à la machine. Cela se fera par des interventions sur les deux plans suivants :

3.1. En instrumentation.- Côtê expêrimental, les efforts vont porter dans un proche avenir sur des modifications de la salle $Y 1$ et du spectromètre MONTPELLIER pour améliorer la résolution du faisceau achromatique et les possibilités de dêtection aux faibles angles. Ce programme englobe aussi l'implantation de l'aimant ASTERIX avec son système de détection sur un support mobile autour de la nouvelle chambre à réaction.

Auprès du séparateur ISOCELE II, le changement automatique des cibles et l'amélioration des sources d'ions sont prévus pour pouvoir fonctionner aux fortes intensités.

3.2. En améliorant l'accêlérateur.- Côté machine il est prévu :

- de rendre l'énergie continûment variable entre les deux points actuels de fonctionnement, ce qui est d'un très grand intérêt pour la physique et principalement pour l'étude des productions de $\pi$ au voisinage du seuil.

- d'assouplir la conduite par une aide informatique de contrôle de nombreux paramètres.

- de mettre au point un nouveau condensateur rotatif permettant un cyclage deux fois plus rapide d'où un gain égal sur l'intensité.

- pour un avenir plus lointain, de réaliser une injection axiale au travers de la culasse, ce qui ouvrirait la voie aux faisceaux de particules polarisées.

- enfin, la création d'un faisceau de neutrons a aussi été envisagêe.

En conolusion, après un an environ de fonctionnement réel, le synchrocyclotron peut être considéré comme une machine stable mais fragile dont les caractéristiques sont, conformes aux prévisions. Le séparateur ISOCELE II est, une grande réussite. Les programmes expérimentaux sont bien amorcés mais ont souffert du manque de régularité du fonctionnement de l'accélérateur. Des résultats originaux ont cependant été obtenus et, après 1 'achèvement des développements prévus, l'ensemble d'orsay sera un excellent outil d'étude de la physique nucléaire à énergie intermédiaire.

\section{REF'ERENCES}

/1/ DEBRAY P., IPN Orsay, communication privée.

/2/ COMPARAT V., et al., IPN Orsay, communication privée.

/3/ WILLIS A. et al., IPN Orsay, communication privée.

/4/ PARIS P., et al., N.I.M. 139 (1976) 251-256. Annuaire 1978 de I'IPN Orsay, N63-64, G12-G23, G31.

/5/ OBERT J., IPN Orsay, communication privée.

/6/ BERG V., Coordinateur Isocele II, IPN Orsay, BP $n^{\circ} 1,91406$ Orsay.

/7/ BERG V. et al., Annuaire 1978 de I'IPN Orsay, N39-N40.

/8/ PARIS P., et al., CSNSM d'Orsay, communication privée.

/9/ BERAUD R., et al., IPN Lyon, communication privée.

/10/ Le BORNEC Y., et al., IPN Orsay, communication privée.

/11/ PILE P.H., et al., Indiana University of Bloomington, communication privêe

PIIE P.H., et al., Phys. Rev. Lett. 42 (1979) 1461 .

/i2/ FRASCARIA Ro, et al., IPN Orsay, communication privée.

113/ LANDAUD G., et al., Laboratoire de Physique Corpusculaire de 1 'Université clermont II, communication privée.

/14/ YUASA T., et al., IPN Orsay, communication privée.

/15/ MARTY N., et aI., IPN Orsay, communication privée.

MORLET M., et al., communication à cette conférence.

/16/ IANGEVIN H., et al., IPN Orsay, communication privée.

/17/ CostILHes J.P., et al., Laboratoire de Physique Corpusculaire de 1 'Université Clermont II, communication privée.

/18/ DIDELEZ J.P., et al., IPN Orsay, communication privée.

/19/ HUSSON J.P., et al., Annuaire 1978 de l'IPN d'orsay, N59 